\title{
Post-Pandemic Digital Education: Investigating Smart Workspaces within the Higher Education Sector
}

\author{
M A Hannan Bin Azhar \\ School of Engineering Technology \& Design \\ Canterbury Christ Church University, UK \\ hannan.azhar@canterbury.ac.uk
}

\author{
Emily Louise Lepore \\ School of Engineering Technology \& Design \\ Canterbury Christ Church University, UK \\ emilylouiselepore@gmail.com
}

\author{
Tasmina Islam \\ Department of Informatics \\ King's College London, UK \\ tasmina.islam@kcl.ac.uk
}

\begin{abstract}
Human collaborations have been challenging with increased dependence on traditional human computer interaction technologies during the pandemic, especially in the education sector. Where conventional learning spaces have not been effective, opportunities have arisen for novel digital innovations to develop a future-proof more realistic learning environment. In the era of postpandemic digital living, a hybrid approach incorporating Internet of Things and augmented reality will transform future classrooms into highly immersive and collaborative learning spaces. This paper examines smart workspaces within the Higher Education sector and presents an energy efficient, future proof, and collaborative framework for smart learning environments.
\end{abstract}

Smart workspace, Higher education, Post-pandemic, Augmented reality, Internet of things.

\section{INTRODUCTION}

During the time of global pandemic, everyone has had to adapt to remote working and remote learning, especially staff and students at higher education institutes have adapted to a new way of working, either remotely or in hybrid mode based on the severity of the state of the pandemic (Baker and Abbo, 2020). Reliance on technology-based solutions to enable education has been vital during this challenging time. 'Smart Education' refers to learning outside a traditional classroom; and is an activity that can be done anywhere and anytime (Bajaj et al., 2018). Abdel-Basset et al., (2018) suggested that use of loT devices within the higher educational sector could inspire smarter lesson plans, enhance access to information and design of more secure campuses. By having smart campuses, it means smart timetabling, parking, energy management and smart learning can all be managed, so students can have improved and elevated experiences (Afshar et al., 2020). Better system management leads to considerable reductions in energy consumption, reducing environmental impact through more efficient lighting, thermal and ventilation control.

A collaborative workspace is all about bringing a team together to pursue a common goal (SpacelQ, 2020) whereas individual workspaces are to do with the space required to complete one's work by themselves. Companies are now embracing innovative space designs like studios, pop-ups and hotdesking instead of traditional offices as these provide good mobility and flow of shared spaces (Khan, 2019). Like the workplaces, smart workspaces in the education sector will allow students to have an enhanced and interactive learning experience, save time and of course, increase productivity (Kaur, 2018). Smartboards and virtual reality are also extremely important in both office and university spaces. Individuals are able to participate in demos as if the product was right in front of them. Augmented reality (AR) should enhance the experience in a learning space as it adds digital elements onto a smart screen (such as Microsoft Hololens, smartphone screen etc.) creating an illusion that holographic content is within the physical world around you (AVRSpot, 2018). AR can provide a view of the real world by using computer-generated information and offers a convenient and intuitive way for users to visualize and interact with loT objects and their data (Dongsik et al., 2019). Current literature (Wired, 2018; Khan, 2019) discusses the need for smart technologies for generic office spaces without detailing how they can be adapted for learning in an education sector. With a preliminary design specification, this paper details the architectural framework for an loT based smart and energy efficient workspace enabling interactive and immersive learning experiences for education. 
In order to justify design choices for smart workspaces in education, a survey was conducted to gather student opinions. Also, examples of existing smart office spaces from literature (e.g. Wired, 2018;Gracia, 2017 etc.) will be given to support arguments. Students currently enrolled in UK universities or those who recently graduated from one were included in the survey. From ten different universities in the UK, 44 responses were received to assess student experiences with higher education technologies. $47.7 \%$ of the participants were male, $52.3 \%$ were female, and they had varying levels of technological proficiency and came from different disciplines (both science and humanities); few had specific learning needs. Participants were asked to rate their opinions on a Likert scale on questions related to the impact smart learning spaces might have on University education, e.g. adaptation of ergonomic factors and automatic climate control around study spaces, flexible learning experiences including interactive remote collaboration and immersive AR based face-to-face learning, privacy issues related to GPS location tracking and health data monitoring through mobile Apps etc. Ethical approval was obtained, and participants gave consent to take part in the study.

The remainder of this paper is organized as follows: Section 2 describes the architectural layers of the proposed framework, Section 3 details the key components, example layouts and benefits of smart workspaces in the higher education sector and finally, Section 4 concludes the paper.

\section{PROPOSED FRAMEWORK}

The following section describes the design framework for a smart workplace within a university setting. The framework is composed of three layers: Internet of Things layer, Edge layer, and Education Solution (Figure 1), which are described below.

\subsection{IoT layer}

The Internet of Things (IoT) layer includes devices such as sensors, actuators and controllers that enable monitoring of equipment in operation and human activity. The layer uses a wireless standard such as ZigBee or Wi-Fi. ZigBee can be more appropriate for constrained loT devices due to its low power consumption and long battery life (ZigBee, 2021). The devices in this layer produce low level time series data such as values of temperature, humidity, heart rate, movement, solar radiation etc. Devices that use the Internet of Things facilitate significant energy savings from efficient usage of lighting, heating, air conditioning, etc., as well as the locating of staff/group members by a real-time locating system. To optimise the efficiency, dimension reduction will be used on the raw data by finding repeated patterns (Muhammad, 2020) before sending it to the edge layer. Data reduction can prevent I/O bottlenecks, as well as dramatically reduce storage, bandwidth, and energy costs.

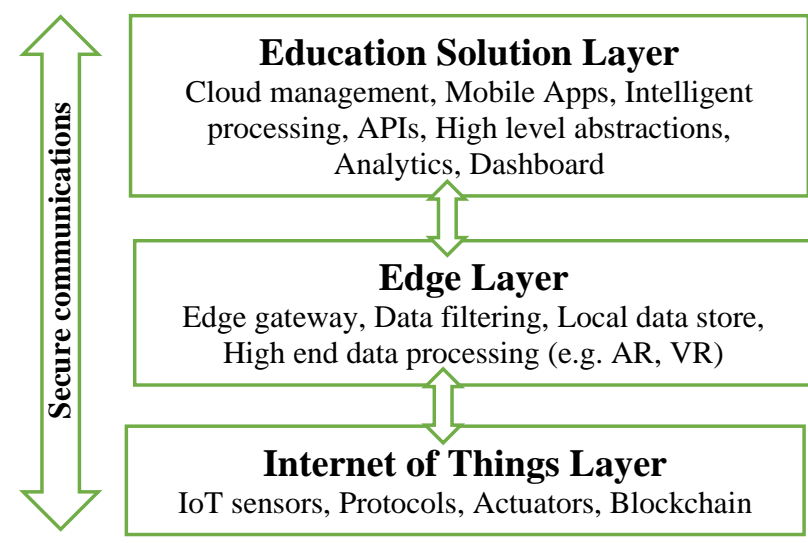

Figure 1: Architecture of smart learning Space

\subsection{Edge layer}

The next layer is the Edge layer, which is the central layer that monitors and updates the technological resources needed to manage the students' activities. The data is filtered in this layer before its being sent to the cloud to be used by the educational applications. Microcomputers such as Raspberry Pis with additional storage capability could be used for low-cost solutions at this layer. In scenarios like VR and AR applications, faster high spec computers and routers can serve as edge gateways for high end data processing and storage. It is possible to use different communications standards depending on the loT scenarios and uses, such as Wi-Fi, Bluetooth, ZigBee, mobile telephony, etc.

\subsection{Education solution layer}

Lastly, there is the education solution layer which is a set of services and Artificial Intelligence (AI) assisted applications to develop intelligent interaction interfaces for development within the education sector. In this layer, the use of APIs allows smart cloud services to be available through web browsers or mobile apps. The aim is to provide edge node management and security, improving energy efficiency within the education workspace. For example, the use of a mobile app to locate or track down staff/group members to increase productivity would be extremely efficient. Apps should also ensure that students are aware of saving energy and changing their behaviours so the resources can be used efficiently.

\subsection{Secure framework}

Data integrity and reliability will be ensured using blockchain loT paradigms (Reyna et al., 2018). For example, use of automatically executed smart contracts at the loT nodes can ensures that no data are modified or corrupted by any other element of 
the network. The proposed distributed architecture for data storage and processing at the edge layer will have an advantage over centralised cloud-based solutions (Manna et al., 2021) as the latter could be vulnerable to denial-of-service (DoS) attacks, where attackers could flood the system and attempt to shut down the server. With the proposed secure architecture, DoS attacks will be prevented, ensuring better response times for systems. Due to the sensitive nature of the data (e.g. student's health data, student id etc.), while data are processed in transit (e.g. over the physical and network layer before reaching the cloud), unsecure communication can be susceptible to intrusions such as the man-inthe-middle attack; it is important that all data transmission should include strong secure encryption techniques and an automated intrusion prevention system should be in place to detect unwanted intrusions and prevent malicious activities.

\section{KEY COMPONENTS AND BENEFITS}

Figure 2 illustrates the key components required to detect and track human presence in a learning space and to measure light levels, temperature, humidity, air quality, etc., to manage energy costs effectively. The example rooms (Figures 3 and 4) incorporate the key components and loT-based Edge Computing services in order to provide faster, more efficient, and secure responses beneficial to student learning. Smart cameras in both rooms can track what is happening by allowing video transmissions for others to view especially those working from home. In both rooms, cloaking the windows is important since it provides transparency and security. As a result, screens cannot be viewed from the outside, which is ideal when confidential information is displayed. To ensure maximum participation, both remote and face-to-face collaborations are incorporated into the design. There are spaces in the room for AR based 3D projections. Students can use smart AR glasses for immersive practical experience when the real work is happening in a remote location. As an example, a virtual demonstration of a human body in a hospital will be transferred to the classroom and medical students wearing AR glasses will be able to see and interact with the demonstration in real time. Further benefits are discussed below.

\subsection{Energy efficiency and sustainability}

The chairs are designed with sensors that adjust automatically so students of different heights can sit ergonomically. In the survey, when students were asked about automatic adaption of ergonomics and the adjustability of the workspace, nearly $90 \%$ said this was beneficial. As standing desks have been scientifically proven to improve productivity, concentration and creativity (Posture Group, 2019), they can be used for both collaborative and individual workspaces. After a certain period of time, smart apps could alert students and staff to stand up, and then the desk will become a standing desk.
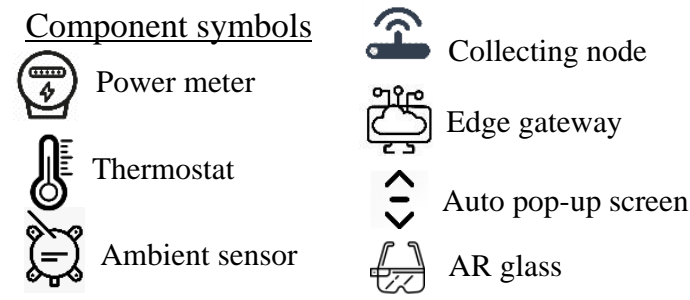

Figure 2: Symbols of key components

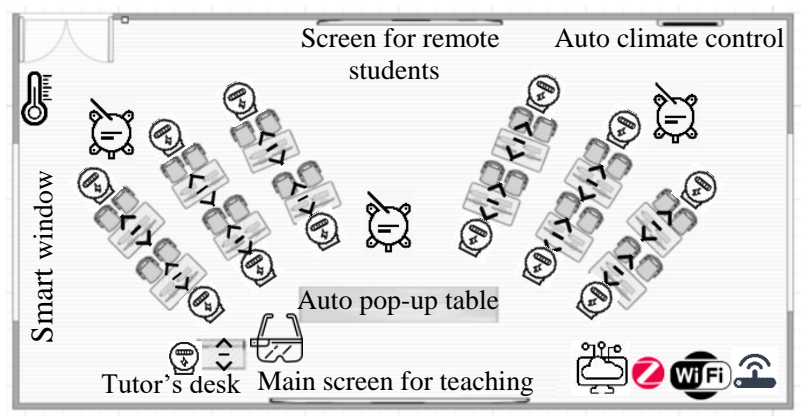

Figure 3: Smart classroom design

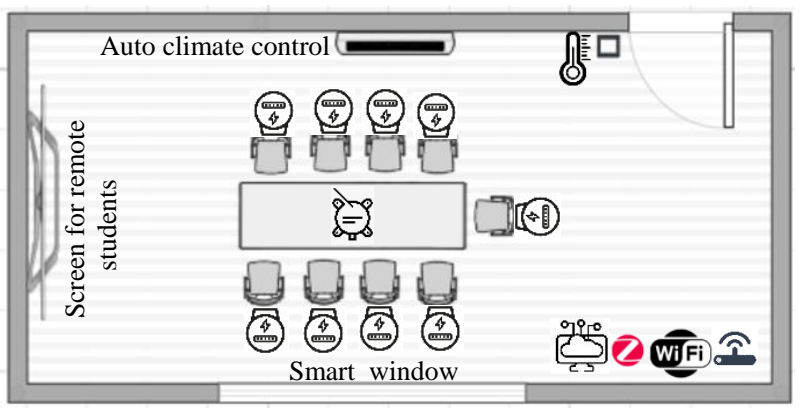

Figure 4: Smart meeting room design

In the survey, when students were asked about automatic climate control, more than $80 \%$ responded that changing the climate depending on their needs would help them focus on studying and increase their productivity. This led to sensors being included in the building design so that temperature, lighting, air quality etc. can be adapted in order for students to get the most out of their studies (Azhar et al., 2017). Edge gateways and collecting nodes will gather information from the workspace, such as temperature, seat settings, fitness levels, etc., to analyse further and make autonomous control of those factors to improve performance and satisfaction during learning. The windows in the rooms (Figures 3 and 4) are smart and regulated automatically to suit best lighting conditions for the intended work. Furthermore, solar panels for water heating and photovoltaic panels on the roof will reduce carbon dioxide emissions significantly. The Edge in Amsterdam, for instance, uses $70 \%$ less power than comparable buildings (Unique Tech News, 2017). A reward point system can be used to 
encourage energy saving habits in students, e.g. obtaining daily power consumption below the previous day's average, taking the stairs instead of the elevator, etc. By collecting enough points, students can get free juice or drinks in the cafeteria.

\subsection{Smart inventory management}

With a mobile app, users can book a meeting room, as shown in Figure 4, almost instantly. Students can book the room immediately after it becomes available online, since availability can be updated in real time. In the survey, when students were asked about the automatic allocation of study rooms based on the schedules, preferences and availability, $82 \%$ agreed that this will increase their productivity by saving time through a transparent autonomous inventory management system. The same concept is demonstrated in an office building in Paris (Majunga Tower, 2021) that allows users to choose a workspace, book a meeting room, control their environment via tablet or phone to support wellbeing and productivity. Also, the Edge in Amsterdam has an loT infrastructure for inventory management, which has significantly increased employee satisfaction and reduced absenteeism by $60 \%$ (British Land, 2017). Furthermore, smart classrooms will be equipped with appropriate sensors to detect component faults (e.g. network printers, routers, etc.) and with $\mathrm{Al}$-assisted software, it will be able to predict system faults and, thus, prevent system downtime. An Italian hospital, for example, used smart fault control to predict $61 \%$ of real faults, including $93 \%$ of overheated appliances (British Land, 2017).

\subsection{Smart wellbeing}

Using wearable sensors in smart gadgets (e.g. smartwatches, mobile phones), it is possible to measure a student's stress level or heart rate and flag up concerns when the condition crosses a threshold. If there is a developing health risk, such as a panic attack, university wellbeing teams can be contacted automatically via text messages. In addition, indoor air quality, light, and noise levels can negatively impact performance and wellbeing. In order to deal with mental health issues, such as depression and dissociation at work, changing colours, circadian lighting systems, and arranging the study spaces according to the time of day can be beneficial.

\subsection{Smart social networking}

Scientists at MIT (British Land, 2017) have developed social physics, which uses data from phones, wearables, and sensors to shed light on how people communicate. An employee's wearable badge can record their whereabouts and who they meet, as well as the nature of the discussions. Students can also take advantage of smart campuses by ordering lunch, booking a meeting room, or tracking staff or other students using mobile apps. Tracking in conjunction with the campus map can serve as a visual guide to find someone. However, despite the fact that tracking a person or measuring health data for wellbeing might be beneficial, survey results show that the majority of students $(79.5 \%)$ were not in favour of personal data collection by smart educational institutions. Student perceptions indicated that personal data collected (e.g. tracking GPS locations, health data from wearable devices, etc.) could easily be misused, leading to more harm than good.

\subsection{Inclusive system}

The survey showed that participants with learning disabilities requested accessibility tools to assist them in learning. In order to ensure that all students have access to the content by default, these resources should be included at the interface level of the smart space. An assisted mobile app can provide resources such as screen readers, tinted screens, options for larger text, text-to-speech software, and blue visors for reading and taking notes. In order to increase the satisfaction level, the default values of the app can be customised to meet the needs of each learner. The app can change the default settings to custom values to the satisfaction level of the learners. With the assistance of Al, app can recommend the optimal settings for each learner.

\section{CONCLUSION}

In this paper, we proposed architectural layers which can be used to implement an loT-based learning space. The survey findings have helped get a better understanding of what students want and how their viewpoints can influence the design of the new smart workspace for learning. The paper described the three-layered architecture of the framework. With the proposed architecture, the computation load is balanced between the cloud and the edge, which results in a reduction of computational capacity, consumption, and hardware resources at the physical or loT layer. The education solution layer on the cloud allows Alassisted applications to identify the optimal settings for information fusion and training. Smarter IOT controlled efficient lighting, thermal and ventilation will lead to considerable reductions in energy consumption to reduce environmental impact meeting low-carbon targets set by the government. In cases where privacy concerns are raised in the survey, the framework justifies the security of the data by proposing the use of the blockchain at the edge and strong encryption. Specifically designed for security and energy efficiency, the framework offers a sustainable learning environment for higher education in the new post-pandemic digital age. 


\section{REFERENCES}

Abdel-Basset, M., Gunasekaran, M., Mohamed, M., and Rushdy, E. (2018) Internet of things in smart educational environment: Supportive framework in the decision-making process, Wiley, Special issue paper, Pages: 1-12.

Afshar Alam, M and Saiyeda, A. (2020) A Cloud Based Solution for Smart Education. Volume 11, Issue 2. Pages 28-29.

AVRSpot (2018) What is Augmented Reality (AR) and how does it work? Available at: https://www.avrspot.com/augmented-reality-arwork/ (Accessed: 28/06/2021).

Azhar, M.H.B., Islam, T. and Alfieri, M. (2017) Effects of students' preferences in use of lighting and temperature on productivity in a university setting. In EAI International Conference on Technology, Innovation, Entrepreneurship and Education (pp. 139-145). Springer, Cham.

Baker, M. D. and Abbo, T., (2020) Working PostPandemic: What Campus Employees Need (Now), Available at: https://er.educause.edu/blogs/2020/5/workingpost-pandemic-what-campus-employees-neednow (Accessed: 28/06/2021).

Bajaj, R and Sharma, V. (2018) Smart Education with artificial intelligence-based determination of learning styles, International Conference on Computational Intelligence and Data Science, ICCIDS, Pages 835-841.

British Land (2017) Smart offices A 2017 vision for the future, Available at: https://officeagenda.britishland.com/assets/pdfs/ smart-offices.pdf (Accessed: 28/06/2021).

Dongsik, J. and Gerard, K. (2019) AR Enabled IoT for a Smart and Interactive Environment: A Survey and Future Directions, Sensors, PMC.

García, Ó., Prieto, J., Alonso, R. and Corchado, J. (2017) A framework to improve energy efficient behaviour at home through activity and context monitoring. Sensors, 17, 1749.

Kaur, D. (2018) How Smart Class Technology is Benefiting Education Sector. Available at: https://www.entrepreneur.com/article/322587

(Accessed: 28/06/2021).

Khan, B. (2019) Trends in Workspace Design with IoT and Tech. Available at: https://www.wrld3d.com/blog/trends-inworkspace-design-with-iot-and-tech/ (Accessed: 28/06/2021).

Manna, S.K., Azhar, M.A.H.B and Sakel, M. (2021) A Smart and Secure IoMT TeleNeurorehabilitation Framework for Post-stroke Patients, International Conference on Industrial
Instrumentation \& Control (ICl2C-2021), to be appeared on Lecture Notes in Electrical Engineering, Springer ISSN: 1876-1100.

Majunga Tower (2021) Eiffage construction's showcase Project of Majunga Tower in Paris, http://www.eiffageconstruction.com/expertise/sh owcase-projects/majunga-tower (Accessed: 28/06/2021).

Muhammad, F.M.M. (2020) Extreme-SAX: Extreme Points Based Symbolic Representation for Time Series Classification. In: Song M., Song IY., Kotsis G., Tjoa A.M., Khalil I. (eds) Big Data Analytics and Knowledge Discovery. DaWaK Lecture Notes in Computer Science, vol 12393. Springer, Cham. https://doi.org/10.1007/978-3030-59065-9_10

Posture Group (2019) Benefits of sit stand desks on your posture and work health \& well-being. Available at: https://www.posturegroup.co.uk/blog/benefits-ofsit-stand-desks-on-your-posture-and-workhealth-and-wellbeing (Accessed: 28/06/2021).

Reyna, A., Martín, C., Chen, J., Soler, E., Díaz, M. (2018) On blockchain and its integration with loT. Challenges and opportunities. Future Gener. Comput. Syst., 88, 173-190.

SpacelQ (2020) What is a Collaborative Workspace, really? Available at: https://spaceiq.com/blog/what-is-a-collaborativeworkspace/ (Accessed: 28/06/2021).

Unique Tech News (2017) Smart office solutions in the world's most sustainable building, Available at: https://uniquetechsolutions.co.uk/smartoffice-solutions-in-the-edge-amsterdam/

(Accessed: 28/06/2021).

Wired (2018) The Workplace of the Future. Available at: https://www.wired.com/brandlab/2018/06/theworkplace-of-the-future/ (Accessed: 28/06/2021).

ZigBee (2021) Why Zigbee? Why should I use Zigbee in my products and services? Available at: https://zigbeealliance.org/why-zigbee/ (Accessed: 28/06/2021). 